Pacific Journal of Mathematics

ON THE GROUPS OF UNITS IN SEMIGROUPS OF

JOHN YUAN 


\title{
ON THE GROUPS OF UNITS \\ IN SEMIGROUPS OF PROBABILITY MEASURES
}

\author{
JOHN YUAN
}

\begin{abstract}
We generalize Pym's decomposition $w=\mu_{E} * w_{H} * \mu_{F}$ of idempotent probability measures to the decomposition $\mu_{E} * \mathscr{H}\left(w_{H}\right) * \mu_{F}$ of the maximal groups of units in semigroup of probability measures on a compact semitopological semigroup. We also prove that $\mathscr{H}(w) \cong \mathscr{H}\left(w_{H}\right) \cong N(H) / H$ algebraically and topologically. With these characterizations, we verify Rosenblatt's necessary and sufficient condition for the convergence of a convolution sequence $\left(\nu^{n}\right)_{n \geqq 1}$ of a probability measure $\nu$ on a compact topological semigroup.
\end{abstract}

1. Introduction. Let $S$ denote a compact semitopological semigroup (i.e., the multiplication is separately continuous) and $(C(S),\|\|)$ the Banach space of all bounded real-valued continuous functions on $S$. Then $M^{b}(S)$ which is defined as the norm dual of $C(S)$ is a Banach algebra under $\|\mu\|=\sup \{|\mu(f)|:\|f\| \leqq 1\}$ and the convolution * which is defined via $\mu^{*} \nu(f)=\int f(x y) \mu(d x) \nu(d y)$ on $C(S)$. Let $P(S)$ be the totality of probability measures on $S$, which consists of all positive measures with norm 1 in $M^{b}(S)$. Then $P(S)$ is a compact semitopological semigroup under ${ }^{*}$ and the weak* topology which is the topology of pointwise convergence on $C(S)$ [4]. If $S$ is topological (i.e., the multiplication is jointly continuous), then $P(S)$ is topological (Prop. 4, [9]).

It is known that every compact semitopological semigroup has a minimal ideal which is not necessarily closed except in the case $S$ is topological [7]. We thus introduce the following definition:

A compact semitopological semigroup is called topologically simple if its minimal ideal is dense in it.

For a subsemigroup $T$ of $S$, we use $E(S)$ and $M(T)$ to denote the totality of idempotents and the minimal ideal in $S$ respectively. For a subsemigroup $A$ of $P(S)$, we write $D(A)=\cup\{\operatorname{supp} \mu: \mu \in A\}$ and $\operatorname{supp} A=$ $\overline{D(A)}$, where supp $\mu$ denotes the support of $\mu$.

In the remainder, $S$ will always denote a compact semitopological semigroup except mentioned especially. 
2. The structure of an idempotent probability measure.

Proposition 2.1. Let $K$ be a compact topologically simple subsemigroup in $S$. Then

1. $E(M(K)) \neq \varnothing$

For $e \in E(M(K))$, we have

2. (a) $\mathrm{H}=e \mathrm{Ke}$ is a compact topological subgroup with identity $e$

(b) $E=E(K e)$ (resp. $F=E(e K))$ is a left (resp. right) zero compact topological subsemigroup

(c) $e E=F e=e, F H=H E=H$ and $F E \subseteq H$

(d) $M(K)=E H F=[E, H, F]$ via

$$
(x, g, y)\left(x^{\prime}, g^{\prime}, y^{\prime}\right)=\left(x, g y x^{\prime} g^{\prime}, y^{\prime}\right)
$$

(e) $K e=(E H F) e=E H$ and $e K=e(E H F)=H F$

3. (a) $P(E)($ resp. $P(F))$ is a left (resp. right) zero compact topological subsemigroup. In particular, $E(P(E))=P(E)$ and $E(P(F))=P(F)$

(b) $\delta_{e}^{*} P(E)=P(F)^{*} \delta_{e}=\delta_{e}$, where $\delta_{e}$ is the point-mass at $e$

(c) $P(F)^{*} P(E) \subseteq P(H)$. In particular,

$$
w_{H} * P(F)^{*} P(E)=P(F)^{*} P(E)^{*} w_{H}=w_{H},
$$

where $w_{H}^{2}=w_{H}$ is the Haar measure on $H$

(d) $P(E) * w_{H} * P(F) \subseteq E(P(S))$.

Proof. 1. (See the proof of 3.4, p. 67, [1]).

2. (See p. 500, [7]; Thm. 2, p. 124, [3]).

3. (a) For $\mu, \nu \in P(E)$,

$$
\mu^{*} \nu(f)=\int f(x y) \mu(d x) \nu(d y)=\int f(x) \mu(d x) \nu(d y)=\mu(f) .
$$

Hence $P(E)$ is left zero. Furthermore, by 2(b) we see that $P(E)$ is a compact topological subsemigroup in $P(S)$.

(b) This follows from 2(c).

(d) Let $\mu=\mu_{E} * w_{H} * \mu_{F} \in P(E) * w_{H} * P(F)$. Then

$$
\mu^{2}=\mu_{E} *\left(w_{H} * \mu_{F} * \mu_{E}\right) * w_{H} * \mu_{F}=\mu_{E} * w_{H} * \mu_{F} .
$$

Lemma A. $\operatorname{supp}\left(\mu^{*} \nu\right)=\overline{(\operatorname{supp} \mu \operatorname{supp} \nu)}$ in $P(S)$.

Proof. [4].

Proposition 2.2. Let $w^{2}=w \in P(S)$. Then 
1. supp $w$ is a compact topologically simple subsemigroup

2. $w=\mu_{E} * w_{H} * \mu_{F}$, where

(a) $H=e(\operatorname{supp} w) e, E=E((\operatorname{supp} w) e)$ and $F=E(e(\operatorname{supp} w))$ for an $e \in E(M(\operatorname{supp} w))$

(b) $\mu_{E} \in P(E)$ with supp $\mu_{E}=E$

(c) $\mu_{F} \in P(E)$ with supp $\mu_{F}=F$

(d) $w_{H}^{2}=w_{H}$ is the Haar measure on $H$

3. $w_{H}=w_{H} * \mu_{F} * \mu_{E}=\mu_{F} * \mu_{E} * w_{H}$

4. $w_{H}=w_{H} * w * w_{H}=w_{H} * \mu_{\dot{F}} * w * \mu_{E} * w_{H}$.

Proof. 1. We refer it to (p. 500, [7]).

2. This is a result of 1 and Proposition 2.1.

3. This is a result of 3(c) in Proposition 2.1.

4. We prove the first equality only. As $e E H F e \subseteq H$,

$$
w_{H} * w * w_{H}=w_{H} *\left(w_{H} * \mu_{E} * w_{H} * \mu_{F} * w_{H}\right) * w_{H}=w_{H}
$$

Proposition 2.3. $E(P(S))=\cup\left\{P(E)^{*} w_{H} * P(F): K\right.$ is a compact topologically simple subsemigroup\}.

3. A characterization of the maximal group of units. For $e \in E(S)$ we denote by $\mathscr{H}(e)$ the maximal group of units with identity $e$ in the compact subsemigroup $e S e$. We will see that $\mathscr{H}(e)$ is in general a locally compact topological subgroup in the relative topology of $S$ and $\mathscr{H}(e)$ is closed and so compact in the case $S$ is topological.

In this section, we maintain that $w^{2}=w=\mu_{E} * w_{H} * \mu_{F}$ is as in Proposition 2.2. In particular, $H$ is a compact subgroup of $\mathscr{H}(e)$.

LEMMA B. $\mathscr{H}(e)$ is a locally compact topological subgroup in the relative topology of $S$. Furthermore, if $S$ is topological, then $\mathscr{H}(e)$ is a closed and hence compact subgroup.

Proof. As $\mathscr{H}(e)$ is a topological subgroup in eSe (Cor. 6.3, pp. $282-283,[6]), \overline{\mathscr{H}(e)}$ is a closed subsemigroup in eSe (3.1, p. 65, [1]). Without losing generality, we may assume that $S=e S e=$ $\overline{\mathscr{H}(e)}$. Suppose that $\mathscr{H}(e)$ is not locally compact. Then $\mathscr{H}(e)$ is not open in $S$. Thus if 0 is an open neighborhood of $e$ in $S$, then $0 \cap(S-\mathscr{H}(e)) \neq \varnothing$, for translation by an element of $\mathscr{H}(e)$ is a homeomorphism of $S$. Now, we choose a relatively compact open neighborhood $U$ of $e$ in $S$. Then $(U \cap \mathscr{H}(e))^{-1}$ is open in $\mathscr{H}(e)$ and contains $e$, so there is an open neighborhood $V$ of $e$ in $S$ so that $V \cap \mathscr{H}(e)=(U \cap \mathscr{H}(e))^{-1}$. Then $U \cap V$ is an open neighborhood of $e$ in $S$ so that $(U \cap V) \cap \mathscr{H}(e)$ is symmetric (i.e., $h \in(U \cap V) \cap \mathscr{H}(e)$ iff $\left.h^{-1} \in(U \cap V) \cap \mathscr{H}(e)\right)$. Since $(U \cap V) \cap(S-\mathscr{H}(e)) \neq \varnothing$, there is an $x$ 
in it. Hence there is a net $\left(h_{\alpha}\right)$ in $\mathscr{H}(e)$ with $h_{\alpha} \rightarrow x$. Since $h_{\alpha}$ is eventually in $U \cap V \subseteq \bar{U}$, there is an $y \in \overline{U \cap V}$ so that $h_{\beta}^{-1} \rightarrow y$ for some subnet $\left(h_{\beta}\right)$. In particular,

$$
x y=\lim h_{\beta} h_{\beta}^{-1}=e
$$

and

$$
y x=\lim h_{\beta}^{-1} h_{\beta}=e .
$$

this contradicts the fact that $x \in S-\mathscr{H}(e)$. Hence $\mathscr{H}(e)$ is locally compact in the relative topology. For the last statement, we refer it to $(2.3$, p. $17,[5])$.

Proposition 3.1. The following statements hold:

1. $\mathscr{H}\left(w_{H}\right)=\left\{w_{H} * \delta_{x}: x \in N(H)\right\}$, where $N(H)$ is the normalizer of $H$ in $\mathscr{H}(e)$ and $\delta_{x}$ are the point-masses

2. The maps $\mathscr{H}(w) \underset{\beta}{\stackrel{\alpha}{\rightleftarrows}} \mathscr{H}\left(w_{H}\right)$ defined via

$$
\alpha(\mu)=\left(w_{H} * \mu_{F}\right) * \mu *\left(\mu_{E} * w_{H}\right)=w_{H} * \mu * w_{H}
$$

and

$$
\beta(\nu)=\mu_{E} * \nu * \mu_{F}
$$

are mutually inverse continuous group-morphisms.

Proof. 1. We prove it in three steps:

(i) $\operatorname{supp} \mu \subseteq e S e$ for all $\mu \in \mathscr{H}\left(w_{H}\right)$.

(ii) Let $\mu \in \mathscr{H}\left(w_{H}\right)$, then there exists a $\nu \in \mathscr{H}\left(w_{H}\right)$ so that $\mu * \nu=$ $\nu * \mu=w_{H}$. Hence for given $a \in \operatorname{supp} \mu$ and $b \in \operatorname{supp} \nu \delta_{a b} * w_{H}=$ $\delta_{b a} * w_{H}=w_{H}$ and thus $a b H=\overline{a b H}=H=\overline{b a H}=b a H$ or $a b=b a g=h$ for some $g, h \in H$ : let $x=h^{-1} a$ and $x^{\prime}=a g h^{-1}$, then $x b=b x^{\prime}=e$ and so $x^{\prime}=e x^{\prime}=(x b) x^{\prime}=x\left(b x^{\prime}\right)=x$. Furthermore,

$$
\mu * \delta_{b}=\left(w_{H} * \mu\right) * \delta_{b}=w_{H} *\left(\mu * \delta_{b}\right)=w_{H}
$$

and so $\mu=w_{H} * \delta_{x}=w_{H} * \delta_{x} * w_{H}$. By (Thm. 1, p. 124, [3]) and Lemma A, we obtain that $H x=\overline{H x}=\overline{H x H}=H x H$. This implies $x \in N(H)$.

(iii) The converse of (ii) follows from the fact that $w_{H} * \delta_{x}=$ $\delta_{x} * w_{H}=w_{H} * \delta_{x} * w_{H}$.

2. We prove it in two steps: 
(i)

$$
\text { (i) } \begin{aligned}
\alpha\left(\mu_{1} \mu_{2}\right) & =w_{H} * \mu_{F} * \mu_{1} * u_{2} * \mu_{E} * w_{H} \\
& =w_{H} * \mu_{F} * \mu_{1} * w * \mu_{2} * \mu_{E} * w_{H} \\
& =w_{H} * \mu_{F} * \mu_{1} * \mu_{E} * w_{H}^{2} * \mu_{F} * \mu_{2} * w_{H} \\
& =\alpha\left(\mu_{1}\right) \alpha\left(\mu_{2}\right), \\
\beta\left(\nu_{1} \nu_{2}\right) & =\mu_{E} * \nu_{1} * \nu_{2} * \mu_{F} \\
& =\mu_{E} * \nu_{1} * w_{H} * \nu_{2} * \mu_{F} \\
& =\mu_{E} * \nu_{1} * \mu_{F} * \mu_{E} * w_{H} * \nu_{2} * \mu_{F} \\
& =\beta\left(\nu_{1}\right) \beta\left(\nu_{2}\right) . \\
\text { (ii) } \quad & \alpha\left(\mu_{E} * \nu * \mu_{F}\right) \\
& =w_{H} * \mu_{F} * \mu_{E} * \nu * \mu_{F} * \mu_{E} * w_{H} \\
& =w_{H} * \nu * w_{H} \\
& =\nu, \\
\beta \circ \alpha(\mu) & =\beta\left(w_{H} * \mu_{F} * \mu * \mu_{E} * w_{H}\right) \\
& =\mu_{E} * w_{H} * \mu_{F} * \mu * \mu_{E} * w_{H} * \mu_{F} \\
& =w * \mu * w \\
& =\mu .
\end{aligned}
$$

Proposition 3.2. The following statements hold:

1. $D\left(\mathscr{H}\left(w_{H}\right)\right)=N(H)$ and $\operatorname{supp}\left(\mathscr{H}\left(w_{H}\right)\right)=\overline{N(H)}$

2. $D(\mathscr{H}(w))=E(N(H)) F=[E, N(H), F]$

3. $\operatorname{supp}(\mathscr{H}(w))=\overline{E(N(H)) F}=\overline{[E, N(H), F]}$.

Proof. 1. This follows from Proposition 3.1. 1.

2. This follows from Proposition 3.1.2 and the above statement.

3. This follows from 2 .

So far, we have only an algebraic characterization of $\mathscr{H}(w)$. In the remainder, we will characterize $\mathscr{H}(w)$ and its subgroups topologically.

Proposition 3.3. The map $\eta: N(H) / H \rightarrow \mathscr{H}\left(w_{H}\right)$ defined via

$$
\eta(x H)=w_{H} * \delta_{x}\left(=\delta_{x} * w_{H}\right)
$$

is a topological isomorphism.

Proof. We observe first that $\eta$ is a well-defined algebraic isomorphism. Hence it remains to show that $\eta$ is an open map. To 
each $f \in C(S), F_{f}(x)=\int f(x y) w_{H}(d y)$ is a bounded continuous function constant on each orbit $x H$ in the compact orbit space $e S e / H$. Without losing generality, we may assume that $e S e=S$. Suppose that $a_{\alpha} H \rightarrow a H$ in $N(H) / H$. Then

$$
\delta_{a_{\alpha}} * w_{H}(f)=F_{f}\left(a_{\alpha} H\right) \rightarrow F_{f}(a H)=\delta_{a} * w_{H}(f) .
$$

Hence $\eta$ is a continuous group-morphism. Suppose that $a_{\alpha} H \nrightarrow \rightarrow a H$. Since $\overline{N(H)} / H$ is compact, there is a subnet $\left(a_{\beta} H\right)$ which converges to a $b H \neq a H$. By Urysohn's Lemma, there is a continuous function $F: S \rightarrow[0,1]$ with $F(a H)=0$ and $F(b H)=1$. Clearly,

$$
\begin{aligned}
\delta_{a_{\alpha}} * w_{H}(f o p) & =F o p\left(a_{\alpha}\right)=F\left(a_{\alpha} H\right) \not \supset F(a H) \\
& =F o p(a)=\delta_{a} * w_{H}(F o p)
\end{aligned}
$$

where $p: S \rightarrow S / H$ is the orbit map. Hence $\eta$ is a topological isomorphism.

The following example shows that not all $\mathscr{H}(w)$ are compact:

EXAMPLE. Let $S=R \cup\{\infty\}$ be the one-point compactification of the additive group of real numbers. Then $S$ is a compact semitopological semigroup and $\mathscr{H}\left(\delta_{0}\right)=\left\{\delta_{x}: x \in R\right\}$ which is not compact.

4. On a limit theorem. Rosenblatt has proved a necessary and sufficient condition for the convergence of a convolution sequence $\left(\nu^{n}\right)_{n \geqq 1}$ of a probability measure $\nu$ on a compact topological semigroup (Thm. 1, p. 152, [8]). We will see one side of his condition is an immediate result of our characterizations of the groups of units.

Proposition 4.1. Let $\nu \in P(S)$. Then $1 / n\left(\nu+\nu^{2}+\cdots+\nu^{n}\right)$ converges to an idempotent probability measure $L(\nu) \in P(S)$ so that

1. $\nu^{m *} L(\nu)=L(\nu)^{*} \nu^{n}=L(\nu)$ for all $m, n \geqq 1$

2. $\operatorname{supp} L(\nu)=\overline{M(T)}$, where $T$ is a closed subsemigroup generated by $\nu$, i.e., $T=\overline{\cup\left\{\operatorname{supp} \nu^{n}: n \geqq 1\right\}}$.

Proof. (See Thm. 3, [2]).

In the remainder, we maintain that $\Sigma(\nu)=\left\{\nu^{n}: n \geqq 1\right\}^{-}, K(\nu)=$ $M(\Sigma(\nu))$ and $L(\nu)=\lim 1 / n\left(\nu+\nu^{2}+\cdots+\nu^{n}\right)=\mu_{X} * w_{G} * \mu_{Y}$. Without losing generality, we may assume that $S$ is generated by $\nu$, i.e., $S=$ $T$. Then $\operatorname{supp} L(\nu)=\overline{M(S)}, G=e S e=e(\operatorname{supp} L(\nu)) e, X=E(S e)=$ $E((\operatorname{supp} L(\nu)) e)=\operatorname{supp} \mu_{X}$ and 


$$
Y=E(e S)=E(e(\operatorname{supp} L(\nu)))=\mu_{Y}
$$

for an $e \in E(M(\operatorname{supp} L(\nu)))$ (cf. 3.5, p. 67, [1]). In particular, $\mathscr{H}(L(\nu))=\mu_{X} *\left\{w_{G}\right\} * \mu_{Y}=\{L(\nu)\}$.

LEMMA C. $K(\nu)$ is a compact commutative topological subgroup in $P(S)$.

Proof. (See the proof of 3.4, p. 67, [1]).

Let $w^{2}=w=\mu_{E}^{\prime} * w_{H} * \mu_{F}^{\prime} \in K(\nu)$. In particular, $K(\nu)$ is a compact subgroup of $\mathscr{H}(w)$. Then.

LEMMA D. The following statements hold:

1. $E(M(\operatorname{supp} w))=E(D(\mathscr{H}(w)))=E(D(K(\nu)))$

2. $D(K(\nu)) \subseteq M(S) \subseteq \operatorname{supp} K(\nu)$. In particular, $\operatorname{supp} K(\nu)=$ $M(S)$

3. $E(M(\operatorname{supp} w)) \subseteq M(S)$.

Proof. 1. This follows from the fact that

$$
E([E, H, F])=[E,\{e\}, F]=E([E, N(H), F])=E(D(\mathscr{H}(w)))
$$

2. As $K(\nu)$ is an ideal in $\Sigma(\nu), D(K(\nu)) D(\Sigma(\nu)) \subseteq D(K(\nu)) \subseteq$ $\operatorname{supp}(K(\nu))$ and so supp $(K(\nu))$ is a closed ideal in $S$ (See 3.1, p. 65, [1]), in particular, $\operatorname{supp}(K(\nu)) \supseteq M(S)$. On the other hand, $D(K(\nu))=$ $M(\operatorname{supp}(K(\nu)))($ See 3.1, p. $65,[1])$ and thus $M(S) \supseteq D(K(\nu))$.

LEMMA E. The following statements hold:

1. $\nu^{*} w=w^{*} \nu \in K(\nu)$

2. $L(\nu)^{*} w=w^{*} L(\nu)=L(\nu)$

3. There exists an $e^{2}=e \in M(\operatorname{supp} w) \cap M(S)$

4. $H=e(\operatorname{supp} w) e \subseteq e S e=G$

5. $E=E((\operatorname{supp} w) e)=E(S e)=X$

6. $F=E(e(\operatorname{supp} w))=E(e S)=Y$

7. $Y X \subseteq H$

8. $w=\mu_{X}^{\prime} * w_{H} * \mu_{Y}^{\prime}$ with $\operatorname{supp} \mu_{X}^{\prime}=X$ and $\operatorname{supp} \mu_{Y}^{\prime}=Y$.

Proof. 1. This follows from the fact that $K(\nu)=M(\Sigma(\nu))$.

2. This follows from Proposition 4.1.

2. This follows from Lemma D.

4. This is trivial.

5. Let $w=\mu_{E}^{\prime} * w_{H} * \mu_{F}^{\prime}$. That $L(\nu)=w * L(\nu)=L(\nu) * w$ implies 


$$
\begin{aligned}
\mu_{X} * w_{G} * \mu & =\left(\mu_{E}^{\prime} * w_{H} * \mu_{F}^{\prime}\right) *\left(\mu_{X} * w_{G} * \mu_{Y}\right) \\
& =\mu_{E}^{\prime} * w_{G} * \mu_{Y} \in P(X) * w_{G} * P(Y) .
\end{aligned}
$$

By Propositions 2.1 and 2.2, $\overline{E G Y}=\overline{X G Y}$ and $E=X$.

6. Similarly.

7. This follows from 5 and 6.

8. This is done in the proof of 2 .

LEMMA F. The following statements are equivalent

1. $\nu^{*} w=w^{*} \nu \neq w$

2. $K(\nu) \neq\{w\}$

3. $w \neq L(\nu)$

4. $H$ is a proper closed normal subgroup in $G$ (i.e., $N(H)=G$ ) so that $G=\overline{\cup\left\{g^{n} H: n \geqq 1\right\}}$ for some $g \in G-H$.

Proof. $1 \Rightarrow 2$. This is trivial.

$2 \Rightarrow 3$. Suppose that $w=L(\nu)$. Then $K(\nu)=\mathscr{H}(L(\nu))=$ $\{L(\nu)\}$. This is a contradiction. Hence $w \neq L(\nu)$.

$3 \Rightarrow 1$. Suppose that $w=w^{*} \nu=\nu^{*} w$. Then

$$
w=w^{*}\left(1 / n\left(\nu+\nu^{2}+\cdots+\nu^{n}\right)=1 / n\left(\nu+\nu^{2}+\cdots+\nu^{n}\right)^{*} w\right.
$$

for all $n \geqq 1$. In particular, $w=w^{*} L(\nu)=L(\nu)^{*} w=L(\nu)$.

$1 \Rightarrow 4$. There is a $g \in N(H)-H$ so that $w * \nu=\mu_{X}^{\prime} *\left(w_{H} * \delta_{g}\right) * \mu_{Y}^{\prime}$. Let $\mathscr{H}(w) \underset{\beta}{\stackrel{\alpha}{\rightleftarrows}} \mathscr{H}\left(w_{H}\right)$ be the mutually inverse continuous morphisms of Proposition 3.2. Then

$$
\begin{aligned}
w^{*} \nu^{n}=\left(w^{*} \nu\right)^{n} & =\left(\beta \circ \alpha\left(w^{*} \nu\right)\right)^{n} \\
& =\beta\left(\left(\alpha\left(w^{*} \nu\right)\right)^{n}\right) \\
& =\beta\left(\left(w_{H} * \delta_{g}\right)^{n}\right) \\
& =\beta\left(w_{H} * \delta_{g^{n}}\right) \\
& =\mu_{X}^{\prime} *\left(w_{H} * \delta_{g^{n}}\right) * \mu_{Y}^{\prime} .
\end{aligned}
$$

Furthermore, $\bigcup_{n \geqq 1}\left(\operatorname{supp} \nu^{n} \operatorname{supp} w\right)=\left(\bigcup_{n \geqq 1} \operatorname{supp} \nu^{n}\right)(\operatorname{supp} w)$ and

$$
\begin{gathered}
\overline{\left(\cup \operatorname{supp} \nu^{n}\right)(\operatorname{supp} w)} \supseteq \overline{\left(\cup \operatorname{supp} \nu^{n}\right)}(\operatorname{supp} w) \\
=S(\operatorname{supp} w)=\overline{(X G Y)} \overline{(X H Y)}=\overline{X G Y}
\end{gathered}
$$


(cf. 3.1, p. 55, [1] for the inclusion). This implies $w^{*} \nu$ generates $\overline{X G Y}$ and thus $\alpha(w * \nu)=w_{H} * \delta_{g}$ generates $G$, i.e., $G=\overline{U\left\{g^{n} H: n \geqq 1\right\}}$. That $N(H)=G$ follows easily.

$4 \Rightarrow 2$. Suppose $K(\nu)=\{w\}$. Then $w=L(\nu)$, in particular, $H=G$.

Proposition 4.2. The following statements are equivalent:

1. $H=G$.

2. $L(\nu)=w$.

3. $K(\nu)=\{w\}$.

4. $w^{*} \nu=\nu^{*} w=w$.

PROPOSITION 4.3. If $\left(\nu^{n}\right)_{n \geqq 1}$ converges, then any statement of Proposition 4.2 holds. The converse holds on compact topological semigroups only.

Proof. The first statement is trivial. For the converse part, we refer to (p. 380, [2]).

THEOREM (Rosenblatt). Let $S$ be a compact topological semigroup generated by $\nu$. Then $\left(\nu^{n}\right)_{n \geqq 1}$ does not converge iff there is a proper closed normal subgroup $H$ of $G$ such that

$$
[X, H, Y] \operatorname{supp} \nu=[X, H g, Y]
$$

for some $g \in G-H$ with $G=\overline{\cup\left\{g^{n} H: n \geqq 1\right\}}$.

Proof. It remains to show the "if" part which we refer to (Thm. 1, p. $152,[8])$.

Acknowledgements. The author wishes to thank Drs. Karl H. Hofmann, John R. Liukkonen and Michael W. Mislove for many helpful suggestions.

\section{REFERENCES}

1. J. F. Berglund and K. H. Hofmann, Compact semitopological semigroups and weakly almost periodic functions, Lecture Notes in Mathematics, 42. Springer, 1967.

2. H. L. Chow, Limit measures on compact semitopological semigroups, Math. Scand., 33 (1973), 375-381.

3. E. Ellis, Locally compact transformation groups, Duke Math. J., 24 (1957), 119-126.

4. I. Glicksberg, Weak compactness and separate continuity, Pacific J. Math., 11 (1961), 205-214.

5. K. H. Hofmann and P. S. Mostert, Elements of compact semigroups, Charles E. Merill Books, Inc., Columbus, Ohio, 1966.

6. J. D. Lawson, Joint continuity in semitopological semigroups, III. Jour. of Math., 18 (1974), 275-285. 
7. J. S. Pym, Idempotent probability measures on compact semitopological semigroups, Proc. Amer. Math. Soc., 21 (1969), 499-501.

8. M. Rosenblatt, Markov Processes, Structure and Asympotic Behavior. Springer, 1971.

9. A. Tortrat, Lois de probabilité sur un space topologique complètement regulier et produits infinits à termes indépendantes dans groupe topologique, Ann. Inst. Henri Poincaré, 1 (1965), 217-237.

Received August 9, 1974 and in revised form March 20, 1975.

National Tsing Hua University,

TAIWAN 300 


\section{PACIFIC JOURNAL OF MATHEMATICS}

\section{EDITORS}

RICHARD ARENS (Managing Editor)

University of California

Los Angeles, California 90024

\author{
R. A. Beaumont \\ University of Washington \\ Seattle, Washington 98105
}

\section{J. DugundII}

Department of Mathematics

University of Southern California

Los Angeles, California 90007

D. Gilbarg and J. Milgram

Stanford University

Stanford, California 94305

\section{ASSOCIATE EDITORS}
E. F. BECKENBACH
B. H. NeumanN
F. WoLF
K. YoshidA

\section{SUPPORTING INSTITUTIONS}

\author{
UNIVERSITY OF BRITISH COLUMBIA \\ CALIFORNIA INSTITUTE OF TECHNOLOGY \\ UNIVERSITY OF CALIFORNIA \\ MONTANA STATE UNIVERSITY \\ UNIVERSITY OF NEVADA \\ NEW MEXICO STATE UNIVERSITY \\ OREGON STATE UNIVERSITY \\ UNIVERSITY OF OREGON \\ OSAKA UNIVERSITY
}

\author{
UNIVERSITY OF SOUTHERN CALIFORNIA \\ STANFORD UNIVERSITY \\ UNIVERSITY OF TOKYO \\ UNIVERSITY OF UTAH \\ WASHINGTON STATE UNIVERSITY \\ UNIVERSITY OF WASHINGTON \\ AMERICAN MATHEMATICAL SOCIETY
}

The Supporting Institutions listed above contribute to the cost of publication of this Journal, but they are not owners or publishers and have no responsibility for its contents or policies.

Mathematical papers intended for publication in the Pacific Journal of Mathematics should be in typed form or offset-reproduced (not dittoed), double spaced with large margins. Underline Greek letters in red, German in green, and script in blue. The first paragraph or two must be capable of being used separately as a synopsis of the entire paper. Items of the bibliography should not be cited there unless absolutely necessary, in which case they must be identified by author and Journal, rather than by item number. Manuscripts, in duplicate, may be sent to any one of the four editors. Please classify according to the scheme of Math. Reviews, Index to Vol. 39. All other communications should be addressed to the managing editor, or Elaine Barth, University of California, Los Angeles, California, 90024.

100 reprints are provided free for each article, only if page charges have been substantially paid. Additional copies may be obtained at cost in multiples of 50.

The Pacific Journal of Mathematics is issued monthly as of January 1966. Regular subscription rate: $\$ 72.00$ a year (6 Vols., 12 issues). Special rate: $\$ 36.00$ a year to individual members of supporting institutions.

Subscriptions, orders for back numbers, and changes of address should be sent to Pacific Journal of Mathematics, 103 Highland Boulevard, Berkeley, California, 94708.

PUBLISHED BY PACIFIC JOURNAL OF MATHEMATICS, A NON-PROFIT CORPORATION Printed at Jerusalem Academic Press, POB 2390, Jerusalem, Israel.

\section{Copyright (C) 1975 Pacific Journal of Mathematics All Rights Reserved}




\section{Pacific Journal of Mathematics

Vol. 60, No. $2 \quad$ October, 1975

Waleed A. Al-Salam and A. Verma, A fractional Leibniz q-formula ........... 1

Robert A. Bekes, Algebraically irreducible representations of $L_{1}(G) \ldots \ldots \ldots \ldots 11$

Thomas Theodore Bowman, Construction functors for topological

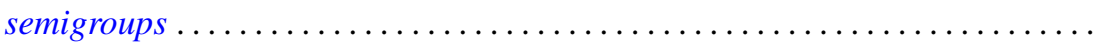

Stephen LaVern Campbell, Operator-valued inner functions analytic on the

closed disc. II .........................................

Leonard Eliezer Dor and Edward Wilfred Odell, Jr., Monotone bases in $L_{p} \ldots \ldots$.

Yukiyoshi Ebihara, Mitsuhiro Nakao and Tokumori Nanbu, On the existence of

global classical solution of initial-boundary value problem for

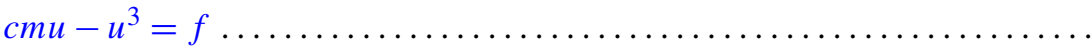

Y. Gordon, Unconditional Schauder decompositions of normed ideals of

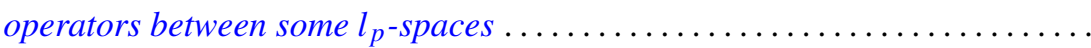

Gary Grefsrud, Oscillatory properties of solutions of certain nth order functional

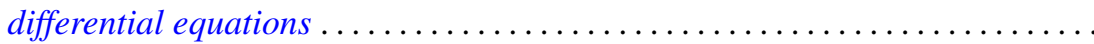

Irvin Roy Hentzel, Generalized right alternative rings ...................

Zensiro Goseki and Thomas Benny Rushing, Embeddings of shape classes of compacta in the trivial range .................................

Emil Grosswald, Brownian motion and sets of multiplicity . .

Donald LaTorre, A construction of the idempotent-separating congruences on a

bisimple orthodox semigroup .

Pjek-Hwee Lee, On subrings of rings with involution ...

Marvin David Marcus and H. Minc, On two theorems of Frobenius ...

Michael Douglas Miller, On the lattice of normal subgroups of a direct

product. .

Grattan Patrick Murphy, A metric basis characterization of Euclidean space

Roy Martin Rakestraw, A representation theorem for real convex functions ....

Louis Jackson Ratliff, Jr., On Rees localities and $H_{i}$-local rings ...

Simeon Reich, Fixed point iterations of nonexpansive mapping . .

Domenico Rosa, $B$-complete and $B_{r}$-complete topological algebras ...

Walter Roth, Uniform approximation by elements of a cone of real-valued

functions ....

Helmut R. Salzmann, Homogene kompakte projektive Ebenen

Jerrold Norman Siegel, On a space between $B H$ and $B_{\infty} \ldots$

235

Robert C. Sine, On local uniform mean convergence for Markov operators

James D. Stafney, Set approximation by lemniscates and the spectrum of an

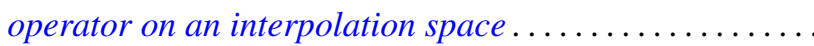

Árpád Száz, Convolution multipliers and distributions .......

Kalathoor Varadarajan, Span and stably trivial bundles ..........

Robert Breckenridge Warfield, Jr., Countably generated modules over

commutative Artinian rings....................... 